\title{
Demand, overcrowding and the pediatric emergency department
}

\author{
Alexander Moylan MBBS, Ian Maconochie MBBS PhD
}

Cite as: CMAJ 2019 June 10;191:E625-6. doi: 10.1503/cmaj.190610

See related article at www.cmaj.ca/lookup/doi/10.1503/cmaj.181426

D emand on pediatric emergency departments is increasing. A linked study provides evidence that overcrowding in Canadian pediatric emergency departments negatively affects the care of both those triaged as requiring urgent care and those requiring less urgent care, by an increased rate of hospital admission and an increased rate of reattendance after discharge, respectively. ${ }^{1}$

Doan and colleagues conducted a retrospective cohort study looking at more than 1.9 million attendances to 8 Canadian pediatric emergency departments over 5 years to determine whether overcrowding in the emergency department is associated with adverse outcomes - defined as hospital admission at the index visit, return to the department or hospital admission within 7 days of discharge from the emergency department, and mortality within 14 days.

For policy-makers in the United Kingdom, this question has already been answered. The first evidence-based principle of the Keogh review of urgent and emergency care services - that "preventing crowding in emergency departments improves patient outcomes" 2 - drove a national effort to reduce emergency department attendances through the reconfiguration and promotion of community services. Although the authors of the linked research found no significant association between emergency department crowding and either hospital admission or mortality after discharge, they did not consider the full range of outcomes examined in other studies, which indicate that quality of treatment, provision of analgesia and patient experience are all negatively affected by overcrowding in the pediatric emergency department. ${ }^{3,4}$

The lack of an increase in hospital admission within 7 days or mortality within 14 days shows an element of resilience among Canadian clinicians and emergency departments. However, Doan and colleagues did observe "an increase in the odds of hospital admission at the index visit with increasing departmental length of stay among the sickest children (Canadian Triage and Acuity Scale [CTAS]) 1-3)." This lowering of the threshold of admission may imply that decision-making became more risk averse during busy periods.

\section{KEY POINTS}

- Attendances at pediatric emergency departments are increasing, despite efforts to tackle overcrowding.

- Several studies have shown that overcrowding in pediatric emergency departments negatively affects the care that patients receive.

- The problem must be tackled at the level of the clinician, the emergency department and the health system, supported by research into evidence-based early warning tools that could improve care delivery in busy environments.

Doan and colleagues' finding that the number of emergency department attendances increased by $27 \%$ over the 5 -year study period is similar to the experience in England, where attendances by those younger than 15 years to emergency departments has increased by $60 \%$ over the past 10 years. ${ }^{5}$ Increasing attendances at pediatric emergency departments pose a challenge to clinicians, emergency departments and health systems. Adjusting to overcrowding by lowering the threshold of admission is not a sustainable response. This need is well recognized by pediatric emergency medicine physicians; a recent survey conducted by the Research in European Paediatric Emergency Medicine network found that fever and risk stratification are research priorities. ${ }^{6}$

Lillitos and colleagues assessed the performance of the Brighton and Children's Observation and Severity Tool (COAST) pediatric early warning scores to predict serious medical and surgical illness and requirement for hospital admission in pediatric patients presenting to the emergency department. ${ }^{7} \mathrm{~A}$ high score was specific but not sensitive for predicting the need for hospital admission and serious medical illness, and performed poorly at predicting serious surgical illness. Cotterill and colleagues looked at the performance of the Pennine Acute Hospitals Trust Paediatric Observation Priority Score (PAT-POPS) and the Manchester Children's Early Warning System (ManChEWS) ${ }^{8}$ in predicting hospital admission within 
72 hours of emergency department attendance. The area under the receiver operating characteristic curve for ManChEWS is 0.67 (95\% confidence interval 0.64-0.70) and for PAT-POPS is 0.72 (95\% confidence interval $0.68-0.75)$. We are currently investigating whether a better-performing early warning score for the pediatric emergency department is available.

Flow and efficiency in the pediatric emergency department are important. Innovations in staffing, nurse-initiated clinical pathways and the addition of observation units ${ }^{9}$ may improve both. At our facility, we have added an observation unit and a colocated urgent care centre. All children are triaged by experienced pediatric nurses and, based on the assessment, a quarter are streamed to this service staffed by primary care physicians.

Access to and parental perception of primary care services are wider systems issues that must be addressed. Children registered at primary care practices that are more easily accessible are $9 \%$ less likely to be brought to the emergency department. ${ }^{10}$ In a survey of parents of children with low-acuity emergency department presentations, only $11 \%$ of whom required emergency department services, Farion and colleagues found that the most common motivations were that emergency departments provide access to everything that the child needs, care is provided by pediatric experts, and the parents trust that everything will be done right. ${ }^{11}$ The authors of the linked study found that overcrowding may adversely affect care that children received, with patients triaged to the least urgent categories (CTAS 4 and 5) more likely to return within 7 days. ${ }^{1}$ This may reflect an unsatisfactory initial visit for the least urgent patients, which may be a result of differing expectations between the parents and the emergency department clinician or emergency departments being poorly set up to meet the needs of these patients.

Despite the efforts described above, there is no evidence that the trend of increasing attendances at pediatric emergency departments is reversing. We must consider the skills that training provides to emergency department physicians and whether there are lessons that could be learned from our colleagues in primary care. The problem must be tackled at the level of the clinician, the emergency department and the health system. Research into tools such as evidence-based early warning systems to support departments in providing safe care in the most demanding situations must support efforts to adapt to this challenge.

\section{References}

1. Doan Q, Wong H, Meckler G, et al.; Pediatric Emergency Research Canada (PERC). The impact of pediatric emergency department crowding on patient and health care system outcomes: a multicentre cohort study. CMAJ 2019;191: E627-35.

2. Transforming urgent and emergency care services in England. Safer, faster, better: good practice in delivering urgent and emergency care. A Guide for local health and social care communities. London (UK): National Health Service England; 2015. Available: www.england.nhs.uk/wp-content/uploads/2015/06/ trans-uec.pdf (accessed 2019 May 13).

3. Sills MR, Fairclough D, Ranade D, et al. Emergency department crowding is associated with decreased quality of care for children with acute asthma. Ann Emerg Med 2011;57:191-200.e1-7.

4. Shenoi R, Ma L, Syblik D, et al. Emergency department crowding and analgesic delay in pediatric sickle cell pain crises. Pediatr Emerg Care 2011;27:911-7.

5. Hospital accident \& emergency activity. Leeds (UK) National Health Service Digital. Available: https://digital.nhs.uk/data-and-information/publications/ statistical/hospital-accident--emergency-activity (accessed 2019 May 15).

6. Bressan S, Titomanlio L, Gomez B, et al. Research priorities for European paediatric emergency medicine. Arch Dis Child 2019 Apr. 25. [Epub ahead of print]. doi:10.1136/archdischild-2019-316918

7. Lillitos PJ, Hadley G, Maconochie I. Can paediatric early warning scores (PEWS) be used to guide the need for hospital admission and predict significant illness in children presenting to the emergency department? An assessment of PEWS diagnostic accuracy using sensitivity and specificity. Emerg Med J 2016;33: 329-37.

8. Cotterill S, Rowland AG, Kelly J, et al. Diagnostic accuracy of PAT-POPS and ManChEWS for admissions of children from the emergency department. Emerg Med J 2016;33:756-62.

9. Barata I, Brown KM, Fitzmaurice L, et al. Best practices for improving flow and care of pediatric patients in the emergency department. Pediatrics 2015;135: e273-83.

10. Cecil E, Bottle A, Cowling TE, et al. Primary care access, emergency department visits, and unplanned short hospitalizations in the UK. Pediatrics 2016; 137:e20151492.

11. Farion KJ, Wright M, Zemek R, et al. Understanding low-acuity visits to the pediatric emergency department. PLoS One 2015;10:e0128927.

Competing interests: None declared.

This article was solicited and has not been peer reviewed.

Affiliation: Department of Paediatric Emergency Medicine, Imperial College Healthcare NHS Trust, UK

Contributors: Both authors wrote the article, gave final approval of the version to be published and agreed to be accountable for all aspects of the work.

Correspondence to: Alexander Moylan, alexander.moylan@nhs.net 